DOI: 10.12957/demetra.2016.17413

\title{
Avaliação da utilização e custo do óleo de soja na produção de arroz e feijão em um restaurante universitário
}

\section{Evaluation of use and cost of soybean oil in the production of rice and beans in a university restaurant}

\author{
Thayana Oliveira Soares' \\ Marlene Azevedo Magalhães Monteiro² \\ Sueli Máximo da Silva ${ }^{3}$ \\ Universidade Federal de Minas Gerais, Curso de \\ Nutrição. Belo Horizonte-MG, Brasil. \\ ${ }^{2}$ Universidade Federal de Minas Gerais, Curso de \\ Nutrição, Departamento de Nutrição, Escola de \\ Enfermagem. Belo Horizonte-MG, Brasil. \\ ${ }^{3}$ Fundação Universitária Mendes Pimental - \\ FUMP. Belo Horizonte-MG, Brasil. \\ Correspondência / Corrrespondence \\ Marlene A. Magalhães Monteiro. \\ Universidade Federal de Minas Gerais - Escola de \\ Enfermagem \\ Av. Prof ${ }^{0}$ Alfredo Balena, 190 - $3^{0}$ andar - Sala \\ 318 - Bairro Santa Efigênia \\ CEP: 30130-100 - Belo Horizonte-MG, Brasil \\ E-mail:marleneaz@enf.ufmg.br
}

\section{Resumo}

Objetivo: O estudo teve como objetivo avaliar a quantidade e o custo do óleo de soja utilizado no preparo do arroz e do feijão, comparando-os com a ficha técnica e com o preconizado pela Organização Mundial da Saúde e pelo Guia Alimentar para a População Brasileira (2006). Métodos: A coleta de dados foi realizada por um período 15 dias em um restaurante universitário por meio da mensuração da diferença entre a quantidade de óleo utilizada nas preparações do almoço com a quantidade total de óleo contido nas latas de 18 litros utilizadas diariamente. Para a comparação dos custos do consumo de óleo entre a quantidade utilizada e a determinada pela ficha técnica, utilizou-se o preço das latas de óleo de 18 litros definido pelo fornecedor do mês. Resultados: Os resultados demonstraram variações nas quantidades de óleo que correspondiam em média a 0,29 $\pm 0,10$ (l) e 0,99 0,42 (l) para feijão e arroz, respectivamente em $10 \mathrm{~kg}$ de grão cru. Tais variações tiveram diferença estatística significativa $(\mathrm{p}<0,05)$ para ambas as preparações. Com relação ao custo no preparo, houve aumento de forma significativa $(\mathrm{p}<0,05)$ ao comparar com os valores da ficha técnica, com uma variação em média de $\mathrm{R} \$ 0,94$ e $\mathrm{R} \$ 1,96$ para a produção de $10 \mathrm{~kg}$ de feijão e arroz, respectivamente. A quantidade de óleo obtida nas porções de arroz $(5,4 \mathrm{ml})$ e de feijão (2,5 ml) também foi elevada se comparada às recomendações diárias de consumo de óleos e gorduras pela Organização Mundial da Saúde (16ml/dia) e pelo Guia Alimentar para a População Brasileira (8ml/dia). Conclusão: Atestou-se a importância da Ficha Técnica de Preparo no controle dos custos na produção de refeições, e que a quantidade de óleo utilizada no preparo é maior do que a recomendada, sendo necessário um programa de capacitação para padronizar a produção das refeições de acordo com a ficha técnica das preparações.

Palavras-chave: Restaurantes; Serviços de Alimentação; Óleo de soja. 


\section{Abstract}

Objective: The study aimed to evaluate the amount and cost of soybean oil used in the preparation of rice and beans, comparing them with the technical specifications and guidelines by the World Health Organization and the Food Guide for the Brazilian Population (2006). Methods: Data collection was carried out for 15 days in a university restaurant by measuring the difference between the amount of oil used in lunch preparations with the total amount of oil contained in cans of 18 liters used daily. For comparing the oil consumption costs between the quantity used and determined by the technical specifications, we used the price of 18-liter oil cans defined by month vendor. Results: The results showed variations in the quantities of oil which corresponded on average $0.29 \pm 0.10$ (l) $0.99 \pm 0.42$ (I) to beans and rice, respectively $10 \mathrm{~kg}$ of raw grain. Such variations were statistically significant difference $(\mathrm{p}<0.05)$ for both preparations. Regarding the cost to prepare, there was increased significantly ( $p<0.05)$ to compare with the data sheet values, with an average variation of $R \$ 0.94$ and $R \$ 1.96$ for the production of $10 \mathrm{~kg}$ of beans and rice, respectively. The quantity of oil obtained in rice portions $(5.4 \mathrm{ml})$ and bean $(2.5 \mathrm{ml})$ was also high as compared to the daily consumption recommendations of oils and fats by the World Health Organization (16ml / day) and the Food Guide for the Brazilian Population ( $8 \mathrm{ml} /$ day). Conclusion: The importance of Preparation Data Sheet in controlling costs in the production of meals, and the amount of oil used in the preparation is greater than recommended, so a training program is necessary to standardize the production of meals according to the data sheet of the preparation.

Key words: Food Service. Soybean oil.

\section{Introdução}

A mudança de hábitos alimentares observada neste século está diretamente relacionada à preferência por uma dieta ocidental que proporcione um maior consumo de alimentos com alto valor energético e ricos em gorduras, ao mesmo tempo que o consumo de frutas, cereais, verduras e legumes se reduz. Essa dieta, aliada a uma diminuição progressiva da atividade física, fator relevante observado na população atual, leva a um aumento da incidência de doenças crônicas não transmissíveis (DCNT), sendo as doenças cardiovasculares, câncer, doença respiratória crônica e diabetes as quatro principais. ${ }^{1,2}$ 
A dieta com um consumo elevado de gorduras, sobretudo saturadas, proporciona aumento na razão LDL-c/HDL-cO, tanto através da ingestão de óleo de soja parcialmente hidrogenado quanto o interesterificado, e está classicamente relacionada com a elevação do LDL-c plasmático e aumento de risco cardiovascular. Sendo assim, a substituição de gordura saturada da dieta por mono e poli-insaturada ou a redução do seu consumo podem ser consideradas estratégias para o melhor controle da hipercolesterolemia, com consequente redução da chance de eventos clínicos. ${ }^{3}$

Apesar dos malefícios de uma dieta inadequada, é importante salientar que o consumo de óleos tem relevância na alimentação humana, pois estes contribuem com a energia necessária para as necessidades nutricionais diárias, contêm vitaminas lipossolúveis, são ricos em ácidos graxos insaturados (ácido oleico, ácido linoleico e alfa-linoleico) e pobres em ácidos graxos saturados. ${ }^{4,5}$ E ainda, proporcionam características sensoriais de maior aceitabilidade pelo consumidor. ${ }^{4,6,7}$

Segundo dados do Instituto Brasileiro de Geografia e Estatística (2008-2009), ${ }^{8}$ estima-se que, nos grandes centros brasileiros, cerca de $30 \%$ das refeições são realizadas fora do domicílio e que a população destina, em média, a parcela de 33,1\% das despesas alimentares com este tipo de refeição. ${ }^{8}$ De acordo com a Pesquisa de Hábitos Alimentares Brasileiros realizada em 2006 em dez cidades brasileiras com mais de um milhão de habitantes cada, com pessoas na faixa etária entre 17 a 65 anos, $94 \%$ dos entrevistados declararam comer arroz e feijão. ${ }^{9}$

A adequação nutricional das refeições, seja no âmbito domiciliar ou na alimentação fora da residência, deve levar em consideração o equilíbrio entre os componentes da refeição que, quando consumidos em excesso, levam a prejuízos à saúde humana.

O papel do nutricionista, ao proporcionar uma dieta equilibrada e balanceada em Unidades de Alimentação e Nutrição (UAN), é de grande impacto, já que sua atuação se estende desde a elaboração de um cardápio que atenda às necessidades nutricionais do indivíduo, até a supervisão da execução adequada do preparo pelos funcionários de acordo com a ficha técnica e avaliação do serviço prestado. ${ }^{5,10}$

Considerando tais atribuições, este trabalho teve como objetivo quantificar a utilização e o custo do óleo de soja utilizado no preparo do arroz e do feijão em uma Unidade de Alimentação e Nutrição institucional.

\section{MATERIAL E MÉTODOS}

O estudo foi realizado em um restaurante universitário situado no município de Belo HorizonteMG, cujo funcionamento ocorre por meio do sistema de subsídio financeiro governamental, com preços diferenciados segundo a categoria socioeconômica dos usuários, a qual exige vinculação do mesmo à universidade ou visitante. ${ }^{11}$ 
O restaurante serve cerca de 3.600 refeições por dia, divididas entre almoço e jantar. O número de usuários varia conforme o dia da semana, com número médio de 2.900 pessoas no almoço e 700 no jantar. O horário de funcionamento é de segunda à sexta-feira (exceto feriados), das $11 \mathrm{~h}$ às $14 \mathrm{~h}$ e das 17h30min às 19h. E aos sábados (exceto feriados), das 11h30min às 13h. Os cardápios tanto para almoço, quanto para o jantar, são constituídos por prato principal (três opções), guarnição, salada, sobremesa, arroz, feijão e refresco. O sistema de distribuição de refeições adotado é o self service, exceto para o prato principal e guarnição, que são porcionados por dois funcionários do restaurante. ${ }^{11} \mathrm{O}$ público-alvo, em sua maioria, é formado por estudantes, e em menor proporção, servidores da instituição de ensino e de órgãos e fundações de apoio da universidade. ${ }^{11}$

A quantificação do óleo de soja nas preparações de arroz e feijão foi realizada por um período 15 dias durante o almoço no mês de maio de 2014. Foram obtidos resultados em triplicata (medições em três dias não consecutivos) para $10 \mathrm{~kg}$ de grão cru e comparados à ficha técnica do restaurante, assim como as recomendações de ingestão diárias de óleos e gorduras pelos órgãos internacionais e nacionais. Além disso, foi estabelecida a variação do custo entre a quantidade de óleo determinada na ficha técnica e a consumida no preparo do arroz e feijão.

Para a pesagem dos grãos crus e cozidos, foi utilizada uma balança digital com capacidade máxima para $500 \mathrm{~kg} / 100 \mathrm{~g}$, uma proveta plástica de $1000 \mathrm{ml}$, com graduações de $5 \mathrm{ml}$ e duas latas de óleo com capacidade de 18 litros, sendo uma vazia para transferência do óleo nas medições e outra identificada para ser utilizada no preparo de arroz ou feijão por dia para o horário do almoço.

Com o intuito de evitar possíveis vieses de mensuração, os funcionários foram instruídos a usarem o óleo da mesma forma de execução anterior ao estudo, mas com as latas identificadas. Após o preparo do arroz e feijão, mediu-se o restante de óleo contido nas latas identificadas com o auxílio da proveta de plástico. Por meio da diferença entre a quantidade restante de óleo contido nas latas e a capacidade de cada lata (18 l), foi possível encontrar a quantidade utilizada nas preparações.

Para os cálculos do rendimento, foi utilizado o Fator de Cocção (Fy), ${ }^{10,12}$ sendo esses valores posteriormente usados para a determinação da quantidade de óleo / porção / preparação em cada dia de mensuração, de acordo com o Guia Alimentar para a População Brasileira ${ }^{13}$ e a Organização Mundial da Saúde (OMS). ${ }^{14}$

A comparação dos dados obtidos com a Ficha Técnica de Preparo (FTP) foi realizada por meio do software Tecfood, da Teknisa ${ }^{\circledR}$, versão 4.09.069, com análise de variância $(\mathrm{p}<0,05)$. E para comparar os custos do consumo de óleo entre a quantidade utilizada e a determinada pela FTP, utilizou-se o preço das latas de óleo de 18 litros definido pelo fornecedor do mês de maio de 2014 $(\mathrm{R} \$ 57,50) .{ }^{15}$

O estudo foi aprovado pelo responsável técnico do restaurante universitário. 


\section{RESULTADOS}

Os resultados descritos nos quadros 1 e 2 demonstram maior utilização de óleo de soja em comparação à FTP, tanto para feijão quanto para o arroz, com diferença significativa $(\mathrm{p}<0,05)$.

Quadro 1. Quantidade de óleo de soja utilizada no preparo de feijão com relação à Ficha Técnica de Preparo do restaurante. Belo Horizonte, 2014.

\begin{tabular}{ccc}
\hline $\begin{array}{c}\text { Quantidade média na Ficha } \\
\text { Técnica de Preparo }(\mathrm{l})\end{array}$ & $\begin{array}{c}\text { Quantidade de óleo de } \\
\text { soja utilizada }(\mathrm{l})\end{array}$ & $\begin{array}{c}\text { Variação entre as duas } \\
\text { medidas }(\mathrm{l})\end{array}$ \\
\hline \multirow{2}{*}{0,23} & 0,63 & 0,40 \\
\cline { 2 - 3 } & 0,42 & 0,19 \\
\hline Média: & 0,54 & 0,31 \\
\hline DP: & 0,53 & 0,30 \\
\hline
\end{tabular}

*Medições em triplicata. Houve diferença significativa estatística $(\mathrm{p}<0,05)$.

Quadro 2. Quantidade de óleo de soja utilizada no preparo de arroz com relação à Ficha Técnica de Preparo do restaurante. Belo Horizonte, 2014.

\begin{tabular}{ccc}
\hline $\begin{array}{c}\text { Quantidade média na Ficha } \\
\text { Técnica de Preparo (l) }\end{array}$ & $\begin{array}{c}\text { Quantidade de óleo de } \\
\text { soja utilizada (l) }\end{array}$ & $\begin{array}{c}\text { Variação entre as duas } \\
\text { medidas (l) }\end{array}$ \\
\hline \multirow{2}{*}{0,26} & 0,70 & 0,44 \\
\cline { 2 - 3 } & 1,05 & 0,79 \\
\hline Média: & 1,00 & 0,74 \\
\hline DP: & 0,91 & 0,66 \\
\hline
\end{tabular}

*Medições em triplicata. Houve diferença significativa estatística $(\mathrm{p}<0,05)$.

Os resultados encontrados demonstraram maior custo na utilização de óleo de soja para o preparo de arroz e feijão em relação à FTP (quadros 3 e 4). Esses resultados tiveram diferença significativa para ambas as preparações $(\mathrm{p}<0,05)$. 
Quadro 3. Custos diários relativos ao uso de óleo de soja para feijão com relação à Ficha Técnica de Preparo do restaurante. Belo Horizonte, 2014.

\begin{tabular}{ccc}
\hline $\begin{array}{c}\text { Custo total médio da Ficha } \\
\text { Técnica de Preparo }\end{array}$ & Custo total do preparo & Variação de custo \\
\hline \multirow{2}{*}{$\$ 0,73$} & $\mathrm{R} \$ 2,01$ & $\mathrm{R} \$ 1,28$ \\
\cline { 2 - 3 } & $\mathrm{R} \$ 1,34$ & $\mathrm{R} \$ 0,61$ \\
\hline Média: & $\mathrm{R} \$ 1,72$ & $\mathrm{R} \$ 0,99$ \\
\hline $\mathrm{DP}:$ & $\mathrm{R} \$ 1,69$ & $\mathrm{R} \$ 0,96$ \\
\hline
\end{tabular}

Quadro 4. Custos diários relativos ao uso de óleo de soja para arroz com relação à Ficha Técnica de Preparo do restaurante. Belo Horizonte, 2014.

\begin{tabular}{ccc}
\hline $\begin{array}{c}\text { Custo total médio da Ficha } \\
\text { Técnica de Preparo }\end{array}$ & Custo total do preparo & Variação de custo \\
\hline & $\mathrm{R} \$ 2,24$ & $\mathrm{R} \$ 1,41$ \\
\cline { 2 - 3 } $\mathrm{R} \$ 0,83$ & $\mathrm{R} \$ 3,35$ & $\mathrm{R} \$ 2,52$ \\
\cline { 2 - 3 } & $\mathrm{R} \$ 3,19$ & $\mathrm{R} \$ 2,36$ \\
\hline Média: & $\mathrm{R} \$ 2,92$ & $\mathrm{R} \$ 2,10$ \\
\hline $\mathrm{DP}:$ & 0,49 & 0,49 \\
\hline
\end{tabular}

A partir dos dados referenciados pelo Guia Alimentar para a População Brasileira ${ }^{13}$ e pela OMS, ${ }^{14}$ foi possível comparar os resultados encontrados da quantidade de óleo de soja contida por porção nas preparações de arroz e feijão (almoço e jantar). Os dados obtidos revelaram uma quantidade elevada de óleo por porção para arroz e feijão, respectivamente, se comparada às recomendações diárias que englobam todas as refeições realizadas ao longo do dia (figura 1). 

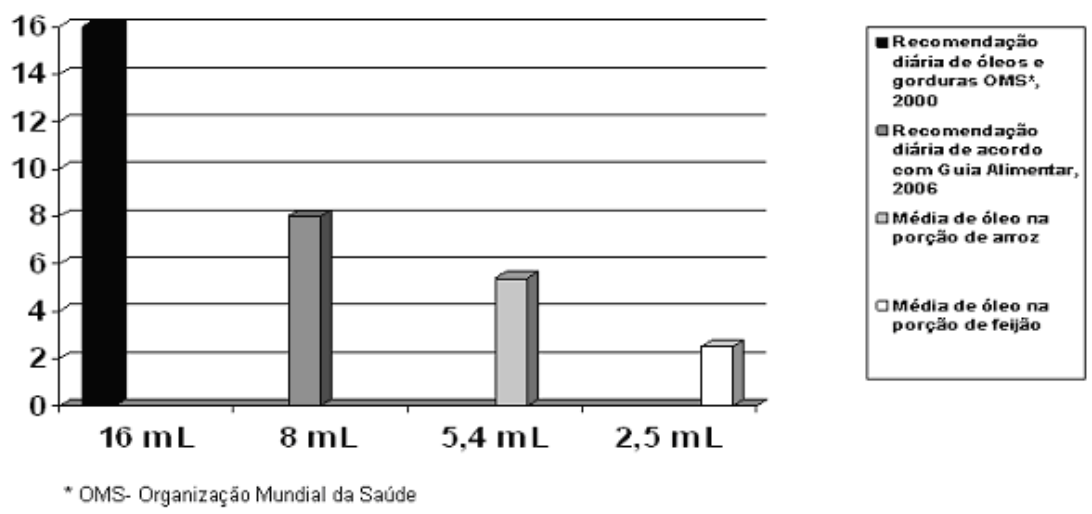

Figura 1. Comparação da quantidade média de óleo de soja nas porções de arroz e feijão em relação à recomendação de consumo diário total de óleos e gorduras. 2000.

\section{DISCUSSÃO}

Foi encontrada diferença significativa $(\mathrm{p}<0,05)$ entre a quantidade média de óleo de soja utilizada nos preparos do feijão e do arroz e a recomendada pela FTP. As variações nas quantidades de óleo de soja corresponderam em média a 0,30 \pm 0,09 (l) para feijão e, 0,66 \pm 0,15 (l) para arroz em 10 kg de grão cru, se comparadas à FTP (tabelas 1 e 2). Apesar de o estudo não ter avaliado a composição centesimal dos lipídeos totais nas preparações, sabe-se que tais valores aumentam consideravelmente na medida em que se aumenta sua quantidade no preparo, devido à sua maior absorção pelos grãos de acordo com o preparo., ${ }^{4}$,

É importante adotar critérios nutricionais no planejamento do cardápio, a fim de se estabelecer o valor energético total das refeições e a distribuição percentual de energia proveniente de proteínas, lipídeos e carboidratos numa refeição, ${ }^{17}$ já que o aumento na quantidade de algum elemento de preparo pode contribuir para um aumento do valor energético inadequadamente.

Com relação aos aspectos nutricionais, o aumento do consumo de óleo de soja pode contribuir para o aumento do consumo total de gordura saturada diária, colaborando com o aumento da adiposidade corporal, além de apresentar forte associação com doenças crônicas degenerativas não transmissíveis. ${ }^{18}$

O ideal é a padronização da quantidade de óleo de soja utilizada para o preparo de arroz e feijão de $8 \%$ e $5 \%$ em relação ao grão cru, respectivamente. ${ }^{4}$ No presente estudo, observou-se que em média 9,1\% foram usados no arroz, e 5,3\% no feijão em relação ao grão cru. Tais valores 
encontram-se acima do ideal sugerido, ${ }^{4}$ tanto para o arroz, que diferiu cerca de $1,2 \%$, quanto para o feijão, com cerca de 0,3\%. Em estudo compararando as características sensoriais (sabor, aroma e textura) nas preparações servidas em um restaurante self servisse, concluiu-se que a padronização para $2 \%$ de óleo no preparo de arroz e feijão não interferia na avaliação e consumo do alimento. ${ }^{4}$

Comparando os resultados obtidos com o preconizado pelo Guia Alimentar para a População Brasileira $^{13}$, observou-se que a quantidade média de óleo $(\mathrm{ml})$ contida em uma porção de arroz (125g - uma colher cheia de servir) e em uma porção de feijão com $50 \%$ de caldo (86 $\mathrm{g}$ - uma concha) representava cerca de 67,5\% (5,4 ml) para arroz, e cerca de 31,2\% (2,5 ml) para feijão do consumo total diário de óleos e gorduras preconizado (8 ml/dia).

Com relação aos dados da OMS, ${ }^{14}$ que preconizam a ingestão de óleos e gorduras de até duas porções diárias (16ml/pessoa - uma colher de sopa cheia), ${ }^{5,14}$ os valores encontrados, em média, para as porções de arroz e feijão correspondem a cerca de 33,7\% (5,4 ml) e 15,6\% (2,5 ml) do recomendado (figura 1 ).

O teor total de óleo ingerido por um indivíduo depende da quantidade consumida e do número de preparações que compõem a refeição. ${ }^{8}$ Sabe-se que a combinação de arroz e feijão está associada a hábitos alimentares tradicionais na população brasileira, mesmo quando a alimentação é realizada fora de casa ${ }^{19}$ tendo forte relação com o consumo maior que uma porção destas preparações nas principais refeições (almoço e jantar). Este fato nos leva a crer que a quantidade de óleo de soja consumida diariamente pelo usuário deste restaurante deve estar acima do recomendado e, por isso, enfatiza-se a necessidade de rever seus procedimentos de produção.

Os resultados encontrados neste estudo são semelhantes ao de Fausto et al., ${ }^{20}$ ao determinarem o perfil e o estado nutricional da clientela e a composição química e nutricional das refeições (almoço e jantar) oferecidas no restaurante universitário da Universidade Estadual Paulista de Araraquara. Os dados indicam porcentuais acima da recomendação para os lipídeos, estes últimos os grandes responsáveis pela elevação do valor energético, não apenas aqueles lipídeos contidos em alimentos de origem animal, como também o lipídeo, adicionado via cozimento e fritura.

Monteiro et al., ${ }^{5}$ ao avaliarem a quantidade de óleo de soja oferecida em um restaurante universitário em Belo Horizonte-MG, observaram que o valor encontrado estava abaixo do preconizado pela OMS. Porém, como este estudo, poderá haver extrapolação da recomendação diária ao considerar as demais refeições do dia.

Outro estudo que analisou a composição nutricional e o custo de matéria-prima de preparações de restaurantes por peso da cidade de Goiânia-GO concluiu que o percentual de sal e óleo nas preparações de arroz branco e feijão de caldo apresentou valores acima do recomendado pela literatura. Diante disso, constatou-se que a ausência de padronização das preparações resultou em diferenças nos custos de produção e na composição nutricional das preparações avaliadas nos restaurantes. ${ }^{21}$ 
Bandoni et al., ${ }^{22}$ ao avaliarem a qualidade da refeição de trabalhadores no município de São Paulo, concluíram que as refeições realizadas no local de trabalho possuem menor densidade energética, maior densidade de fibras e maior participação de hortaliças, frutas e leguminosas, se comparadas às realizadas no domicílio. Por outro lado, as refeições realizadas em restaurantes comerciais resultaram em consumo superior de açúcares e doces e óleos e gorduras, quando comparadas àquelas realizadas no domicílio.

Bezerra e colaboradores,$^{23}$ ao analisarem as características do consumo de alimentos fora do domicílio no Brasil, observou-se que os grupos de alimentos com maior percentual de consumo fora de casa foram bebidas alcoólicas, salgadinhos fritos e assados, pizza, refrigerantes e sanduíches, ou seja, predominância de alimentos de alto valor energético e pobre conteúdo nutricional.

Os parâmetros avaliados neste estudo permitem enfatizar a importância da utilização e padronização da FTP como um instrumento de controle que beneficia todo o processo de produção, pois facilita o trabalho do responsável técnico, permite controlar o valor nutricional das refeições fornecidas e representa um apoio operacional pelo qual se faz o levantamento dos custos. ${ }^{24,25}$ Sendo assim, possibilita a padronização das preparações, fator importante no controle das quantidades de ingredientes utilizado no preparo das refeições. ${ }^{24}$ Além dos aspectos nutricionais, a redução do uso de óleo de soja nas preparações, a fim de se adequar ao preconizado na FTP do restaurante, contribui também para a redução dos custos.

Observou-se variação dos custos com relação à FTP no preparo de feijão e arroz, em média, de $\mathrm{R}$ \$ 0,96 e R \$2,10 para a produção de $10 \mathrm{~kg}$ de alimento cru. Os valores obtidos do custo no preparo corresponderam a 2,3 e 3,5 vezes a mais que o custo obtido pela FTP para feijão e arroz, respectivamente (tabelas 3 e 4), com diferença significativa $(p<0,05)$ para ambos. Não foram encontrados na literatura dados comparativos com relação aos custos da utilização do óleo de soja por preparação.

Ressalta-se, a partir desta análise, a importância da FTP em unidades produtoras de refeições. A principal limitação deste estudo refere-se à avaliação da utilização de óleo de soja no preparo apenas para arroz e feijão.

\section{CONCLUSÃO}

Concluiu-se, portanto, que a quantidade de óleo de soja utilizada no preparo do arroz e do feijão encontra-se elevada, de acordo com os dados da FTP do restaurante e com as recomendações da OMS e do Guia Alimentar para a População Brasileira. Além disso, apesar de a média de oferta do óleo de soja do restaurante universitário estar abaixo do preconizado pela OMS, tal valor pode não refletir a ingestão real do seu usuário, em virtude das demais refeições realizadas ao longo do dia e do consumo de outras fontes de gorduras. 
Também foi possível constatar que a diminuição da quantidade de óleo favorece a redução de custos mensais com a compra deste ingrediente pelo restaurante.

Recomenda-se capacitar os funcionários desse restaurante para enfatizar a importância da utilização da FTP, como forma de promoção da saúde para todos os seus usuários.

\section{REFERÊNCIAS}

1. Duncan BB, Chor D, Aquino EML, Bensenor IM, Mill JG, Schmidt MI, et al. Doenças crônicas não transmissíveis no Brasil: prioridade para enfrentamento e investigação. Rev. Saúde Publica 2012; 46(Suppl 1):126-134.

2. Mariath AB, Grillo LP, Silva RO, Schmitz P, Campos IC, Medina JRP, et al. Obesidade e fatores de risco para o desenvolvimento de doenças crônicas não transmissíveis entre usuários de unidade de alimentação e nutrição. Cad. Saúde Pública 2007; 23(4):897-905.

3. Santos RD et al. Sociedade Brasileira de Cardiologia. I Diretriz sobre o consumo de Gorduras e Saúde Cardiovascular. Arq. Bras. Cardiol. 2013; 100(1 Supl.3):1-40.

4. Amorim MMA, Junqueira RG, Jokl L. Consumo de óleo e gordura nas preparações do almoço self service. Alim. Nutr. 2010; 21(2):217-223.

5. Monteiro MAM, Calixto CFS, Azevedo JFM, Schaefer MA. Avaliação da quantidade de óleo de soja em refeições oferecidas em um restaurante universitário. Demetra: Alimentação, Nutrição e Saúde 2013; 8(1):53-61.

6. Cella RCF, Regitano-D’arce MB, Spoto MHF. Comportamento do óleo de soja refinado utilizado em fritura por imersão com alimentos de origem vegetal. Cienc. Tecnol. Alim. 2002; 22(2):111.

7. Jorge N, Janieri C. Avaliação do óleo de soja utilizado no restaurante universitário do IBILCE/ UNESP. Alim. Nutr. 2004; 15(1):11-16.

8. Instituto Brasileiro de Geografia e Estatística. Pesquisa de orçamentos familiares 2008-2009: despesas, rendimentos e condições de vida. Rio de Janeiro: IBGE; 2010. [acesso em: 02 nov. 2012]. Disponível em: http://www.ibge.gov.br/home/estatistica/populacao/condicaodevida/pof/2008_2009/ POFpublicacao.pdf.

9. Barbosa L. Feijão com arroz, arroz com feijão: o Brasil no prato dos brasileiros. Horiz. Antrop. 2007; 13(28):87-116.

10. Amorim MMA, Junqueira RG, Jokl L. Adequação nutricional do almoço self service de uma empresa de Santa Luzia, MG. Rev. Nutr. 2005; 18(1):145-156.

11. Fundação Mendes Pimentel. Alimentação. Restaurantes universitários [Internet]. Belo Horizonte: Fump, UFMG. [acesso em: 13 maio 2014]. Disponível em: http://www.fump.ufmg.br/conteudo. aspx? pagina $=137$

12. Costa V, Rosa MC. Medidas caseiras. Florianópolis: PET Nutrição, UFSC; 1997. [apostila] 
13. Brasil. Ministério da Saúde. Secretaria de Atenção à Saúde. Coordenação Geral da Política de Alimentação e Nutrição. Guia alimentar para a população brasileira: promovendo a alimentação saudável. Brasília: Ministério da Saúde; 2006.

14. World Health Organization. Obesity: preventing and managing the global epidemic. Report of a WHO consultation on obesity. Geneva: WHO; 2000.

15. Fundação Mendes Pimentel. Restaurantes universitários terão reajuste [Internet]. Belo Horizonte: Fump, UFMG; 2013. [acesso em: 13 maio 2014]. Disponível em: https://www.ufmg.br/online/ arquivos/2005/01/restaurantes_universitrios_ter.shtml

16. Silva MR, Miranda MZ, Silva PRM, Xavier SC. Absorção de óleo de soja e sódio em arroz e feijão preparados. Pesq. Agrop. Trop. 2004; 34(1):21-27.

17. Oliveira RB, Guaglianoni DG, Demonte A. Perfil do usuário, composição e adequação nutricional do cardápio oferecido em um restaurante universitário. Alim. Nutr. 2005; 16(4):397-401.

18. World Health Organization. Diet, nutrition and the prevention of chronic diseases. Geneva: WHO; 2003.

19. Rodrigues AGM, Proença RPC, Calvo MCM, Fiates GMR. Perfil da escolha alimentar de arroz e feijão na alimentação fora de casa em restaurante de bufê por peso. Cienc. Saúde Colet. 2013; 18(2):335-346.

20. Fausto MA, Ansaloni JA, Silva ME, Garcia Júnior J, Dehn AA, Césa Tb. Determinação do perfil dos usuários e da composição química e nutricional da alimentação oferecida no restaurante universitário da Universidade Estadual paulista, Araraquara, Brasil. Rev. Nutr. 2001; 14(3):171-176.

21. Cabral HCC, Morais MP, Carvalho ACMS. Composição nutricional e custo de preparações de restaurantes por peso. Demetra: Alimentação, Nutrição e Saúde 2013; 8(1):23-38.

22. Bandoni DH, Canella DS, Levy RB, Jaime PC. Alimentação dentro ou for a do domicílio: análise da qualidade da refeição segundo o local de realização. Rev. Nutr. 2013; 2(6):625-632.

23. Bezerra IN, Souza AM, Pereira RA, Sichieri S. Consumo de alimentos fora do domicílio no Brasil. Rev. Saúde Pública 2013; 47(1Supl):200S-211S.

24. Araújo VMC, Borgo LAB, Montebello NP, Botelho RPB. Alquimia dos alimentos. Brasília: Senac; 2008. p. 209-213.

25. Machado M, Martins MAA, Andrade GQP, Kraemer FB. A qualidade nutricional e sensorial de preparações proteicas de unidades de alimentação e nutrição escolares: uma avaliação pelo método AQNS. Demetra: Alimentação, Nutrição e Saúde 2012; 7(3):145-156.

Recebido: 15/7/2015

Revisado: 18/9/2015

Aceito: 16/1/2016 
Demetra: AlimentaÇÃo, NUTRIÇÃo \& SAÚdE

110 DemerRa; 2016; 11(1); 99-110 